\title{
Biologically Weighted Quantities in Radiotherapy: an EMRP Joint Research Project
}

\author{
Hans Rabus ${ }^{1,}$ a, Hugo Palmans ${ }^{2,3}$, Gerhard Hilgers ${ }^{1}$, Peter Sharpe ${ }^{2}$, Massimo Pinto $^{4}$, Carmen Villagrasa ${ }^{5}$, Heidi Nettelbeck ${ }^{1}$, \\ Davide Moro ${ }^{6}$, Andrea Pola ${ }^{7}$, Stanislaw Pszona ${ }^{8}$, Pedro Teles ${ }^{9}$ \\ ${ }^{1}$ Physikalisch-Technische Bundesanstalt (PTB), Braunschweig, Germany \\ ${ }^{2}$ National Physical Laboratory (NPL), Teddington, UK \\ ${ }^{3}$ EBG MedAustron GmbH, Wiener Neustadt, Austria \\ ${ }^{4}$ Istituto Nazionale di Metrologia delle Radiazioni Ioniszanti (ENEA-INMRI), Via Anguillarese , 301, 00123 Santa Maria \\ di Galeria RM, Italy \\ ${ }^{5}$ Institut de Radioprotection et de Sûreté Nucléaire (IRSN), Fontenay-aux-Roses, France \\ ${ }^{6}$ Istituto Nazionale di Fisica Nucleare (INFN), Legnaro, Italy \\ ${ }^{7}$ Politecnico di Milano (PoliMi), Milan, Italy \\ ${ }^{8}$ National Centre for Nuclear Research (NCBJ), Otwock-Swierk, Poland \\ ${ }^{9}$ Universidade Tecnica de Lisboa, Instituto Tecnológico e Nuclear (IST-ITN), Sacavém, Portugal
}

\begin{abstract}
Funded within the European Metrology Research Programme (EMRP) [1], the joint research project "Biologically weighted quantities in radiotherapy" (BioQuaRT) [2] aims to develop measurement and simulation techniques for determining the physical properties of ionising particle tracks on different length scales (about 2 $\mathrm{nm}$ to $10 \mu \mathrm{m}$ ), and to investigate the correlation of these track structure characteristics with the biological effects of radiation at the cellular level. Work package 1 develops micro-calorimeter prototypes for the direct measurement of lineal energy and will characterise their response for different ion beams by experiment and modelling. Work package 2 develops techniques to measure particle track structure on different length scales in the nanometre range as well as a measurement device integrating a silicon microdosimeter and a nanodosimeter. Work package 3 investigates the indirect effects of radiation based on probes for quantifying particular radical and reactive oxygen species (ROS). Work package 4 focuses on the biological aspects of radiation damage and will produce data on initial DNA damage and late effects for radiotherapy beams of different qualities. Work package 5 provides evaluated data sets of DNA cross-sections and develops a multi-scale model to address microscopic and nanometric track structure properties. The project consortium includes three linked researchers holding so-called Researcher Excellence Grants, who carry out ancillary investigations such as developing and benchmarking a new biophysical model for induction of early radiation damage and developing methods for the translation of quantities derived from particle track structure to clinical applications in ion beam therapy.
\end{abstract}

\section{Introduction}

In cancer treatment using ionising radiation (radiotherapy), dosage is quantified by the absorbed dose to water expressed in the derived SI unit gray. Several novel and advantageous radiotherapy modalities like proton and ion beams require an additional weighting factor to account for increased tumour cell mortality for a given absorbed dose (as compared to a high-energy photon beam). The growing use of radiotherapy modalities whose biological effect differs from that of conventional highenergy photon beams raises the need to establish a new dosimetric concept which allows a transparent separation of the physical processes (dependent on modality) from the biological ones (independent of modality) in order to ensure consistency of dose prescription across the different techniques. This will allow for better comparisons and evaluations of clinical results using different and new radiation therapies, which in turn are expected to result in optimised treatment planning through a better understanding and choice of radiation qualities.

Radiation quality is conventionally characterised by specifying the energy spectrum of the ionising particles outside the human body. An alternative characterisation of radiation quality for charged particles is the linear energy transfer (LET), i.e. the average energy loss of the particle per path length [1]. While already accounting for the interaction of the radiation within the human body, LET has the same shortcoming as absorbed dose, namely that it is an average value. More precisely, both quantities are

\footnotetext{
${ }^{\text {a }}$ Corresponding author: hans.rabus @ptb.de
} 
expectation values of statistical distributions originating from the stochastic nature of radiation interaction which is governed by the so-called track structure.

For a single ionising particle, the track is the entirety of all loci of interactions, the types of interactions and related and energy transfers occurring at these points and the induced chemical consequences of the radiation interaction such as bond breaks in biomolecules (e.g. the DNA) or water molecules. The latter effect, water radiolysis, leads to the formation of radicals that, in turn, can react with biomolecules to produce the so-called indirect damage (as opposed to direct damage induced by the direct interaction of ionising particles with biomolecules) [4].

The physical part of the aforementioned radiation weighting factors accounting for the different biological effectiveness of different radiation qualities is determined by the microscopic details of the particle track structure. Microdosimetry [5] and nanodosimetry [6,7] have been developed to quantify the properties of the physical part of track structure in terms of stochastic microscopic quantities related to the transfer of energy to cells in tissue and the ionisation in chromosomes or DNA. For instance, one such quantity in microdosimetry is the lineal energy, defined as the ratio of the energy imparted to a particular micrometric target and the mean chord length of a passing particle track within the target volume. For nanometric targets, on the other hand, applying a mean energy required for producing an electron-ion pair for converting ionisation to energy is no longer possible. Hence, in nanodosimetry quantities like the ionisation cluster size are used, defined as the number of ionisations produced within a nanometric target by a particular track.

In both, micro- and nanodosimetry, distinct radiation qualities differ in the probability distributions that are obtained for respective stochastic quantities when measuring track structure. However, neither of these two microscopic dosimetry methods on its own entirely can describe the biological effects as function of the distribution of energy or ionisation depositions. In addition, present day microdosimeters and nanodosimeters measure ionisation in gases or semiconductors that are not necessarily representative of the energy deposition and ionisation in tissue.

\section{The BioQuaRT project}

Given the complexity of the initiation and occurrence of biological processes on various scales which depend on both ionisation and non-ionisation events, a multi-scale approach is therefore needed to lay the foundation for new physical quantities relating track structure to relative biological effectiveness in proton and ion beam therapy. Realising this approach is the purpose of the joint research project BioQuaRT (Biologically Weighted Quantities in Radiotherapy) which started in June 2012 and runs for three years.

Within BioQuaRT measurement and simulation techniques for determining the physical properties of ionising particle track structure on different length scales from about $2 \mathrm{~nm}$ (diameter of DNA double helix) to about
$10 \mu \mathrm{m}$ (diameter of cell nucleus) are developed to allow a multi-scale characterisation of the radiation qualities used in ion beam therapy. A comprehensive multi-scale simulation tool is being developed that will include data for radiation interaction cross sections with DNA and the production rates of radical species, where both kind of data are experimentally determined within the project. Furthermore, it will be investigated at the cellular level how these track structure characteristics correlate with the biological effects of radiation such as to benchmark an extension of the multi-scale simulation predicting biological consequences of track structure.

The project draws upon the metrological expertise of several European National Metrology Institutes (NMIs) and Designated Institutes (DIs, according to the rules of the mutual recognition arrangement, MRA), which maintain leading-edge expertise in micro-calorimetry, nanodosimetry, chemical probes, and Monte Carlo track structure codes as well as in radiobiology (biological experiments needed to validate some predictions of the physical models). The project consortium is reinforced by so-called Researcher Excellence Grants (REGs) that have been awarded to three researchers at institutes outside the $\mathrm{NMI} / \mathrm{DI}$ community. In addition, the project benefits from interaction with 13 associated collaborators [2].

\section{Status of the individual work packages}

\subsection{Microdosimetry}

Energy deposition at the micrometre scale determines both direct and indirect radiation damage which occurs by reactions of the DNA with radical species formed by water radiolysis. Traditional microdosimeters like tissueequivalent proportional counters (TEPCs) and silicon devices primarily measure ionisation distributions. These are then converted to imparted-energy based microdosimetric quantities by multiplication with the mean energy required to produce an electron-ion pair. However this proportionality factor between ionisation and energy distributions depends on the particle kind and its energy. Therefore, when considering only a single constant factor, the conversion from ionisation to energy distribution is subject to a significant systematic uncertainty in mixed-radiation fields.

Further systematic uncertainties are linked to the fact that proportional counters measure ionisation in a (tissue equivalent) gas whereas the radiation interaction in biological systems occurs in the condensed phase. On the other hand, silicon microdosimeters measure ionisation in the condensed phase, but in a material that is not tissue equivalent. To determine the required corrections for condensed phase effects or tissue non-equivalence, a micro-calorimeter with carbon absorber is developed that will allow the direct measurement of lineal energy in tissue equivalent material.

The micro-calorimeter under development $[8,9]$ is based on the technology of an Inductive Superconductive Transition Edge Detector (ISTED) [10]. The detector is based on the principle of a DC Superconducting QUantum Interference Device (SQUID) and consists of a 
superconducting loop, interrupted by two micro-bridge junctions, around a mixed superconducting and tissueequivalent absorber. This detector is extremely sensitive to changes in magnetic field, via the SQUID, with the radiation absorbing element located within the loop.

Energy imparted to the absorber causes Cooper pairs in the superconductor to break resulting in a change in inductance which can be measured by the SQUID loop. Since the binding energy of the Cooper pairs is of a few $\mathrm{meV}$ the detector has the potential to be up to 1000 times more sensitive than a semi-conductor detector and 10000 times more sensitive than a tissue-equivalent proportional counter (TEPC).
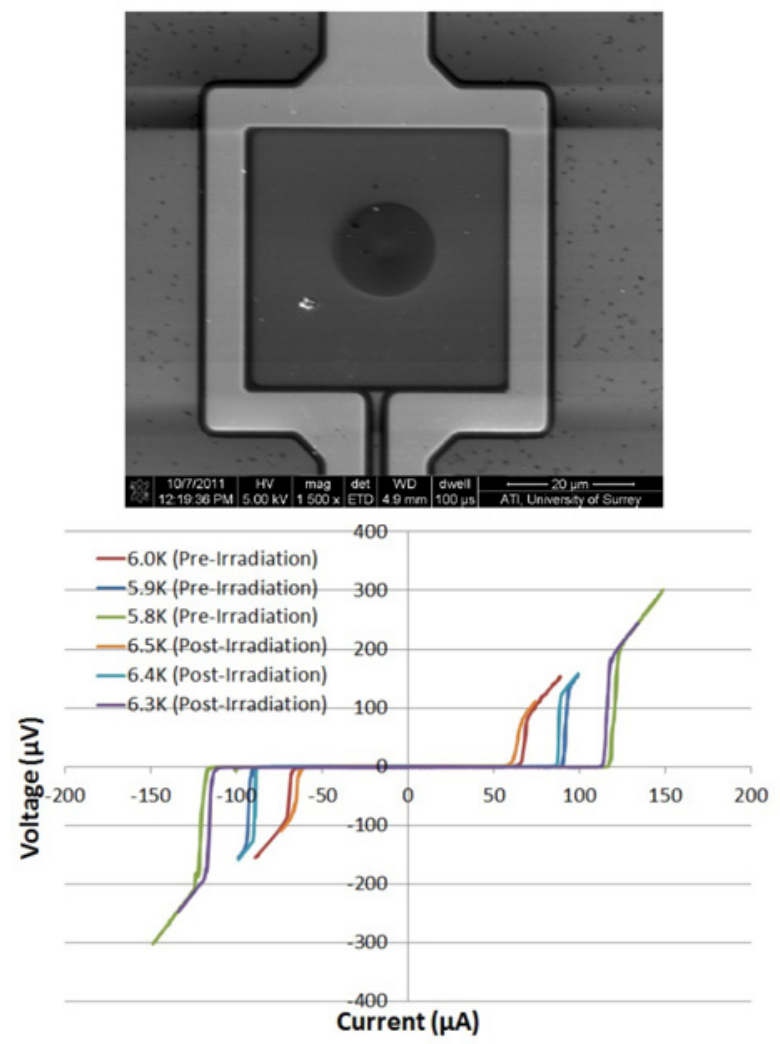

Figure 1. Top: electron micrograph of one of the prototype devices showing the SQUID loop and the absorber (about $15 \mu \mathrm{m}$ diameter) deposited in the centre. Bottom: current-voltage characteristic for one of the prototype SQUIDS before and after irradiation. (Figures reproduced from reference [9]).

A number of prototype detectors with absorber diameters between 5 and $25 \mu \mathrm{m}$ has been produced (an example is shown in figure 1) and electronically characterised and a preliminary radiation experiment was performed to test their radiation hardness. As an example, figure 1 shows the current-voltage characteristics of one of the SQUIDs before and after irradiation. A shift in the temperature observed to obtain the same characteristic was explained as a change in heat sinking of the cables rather than a physical change of the device [9].

Apart from the prototype production and electronic testing of these micro-calorimeters, work performed so far has involved Monte Carlo particle transport simulations and heat transfer simulations. The former is performed using Geant4 to aid the design of the prototypes [9] and will play an essential role in deriving microdosimetric spectra from the measured signal distributions. For the heat transfer simulations finite element calculations using Comsol Multiphysics software were executed to characterise the temperature equilibration within the absorber and heat losses to the silicon substrate [11].

To validate the operation of the micro-calorimeters and, in the future, to investigate the assumption made in ionisation-based microdosimeters that there is a proportionality between ionisation and energy deposition, comparisons will be performed with a silicon-microtelescope [12,13] and a mini-TEPC [14] that both have been tested extensively in proton beams $[15,16]$.

\subsection{Nanodosimetry}

In nanodosimetry, the ionisation component of the particle track structure is characterised by the relative frequency distribution of the ionisation cluster size. Ionisation cluster size thereby means the number $v$ of ionisations generated in a target volume by a primary particle and its secondary electrons. To date, three types of nanodosimeter have been developed that measure frequency distribution of ionisation cluster size in a gas, where the respective target volumes correspond to different sizes of nanometric targets in biological matter. The Jet Counter at NCBJ detects positive ions produced by primary particles passing centrally through a pulse of nitrogen gas simulating biological target sizes of about $2 \mathrm{~nm}$ and $20 \mathrm{~nm}$ by adjusting the density of molecules [17, 18]. The StarTrack Counter at INFN detects electrons generated in a sensitive volume of propane equivalent to a $20 \mathrm{~nm}$ biological target intersected by the primary particle track at a defined distance from the target centre [19, 20]. The PTB Ion Counter also detects positive ions produced in a gas target corresponding to a target of about $2 \mathrm{~nm}$ in diameter [21, 22].

The equivalence of the ionisation cluster size distribution measured in these devices and the corresponding nanometric targets in condensed matter, such as liquid water or biological tissue, is based on a density scaling approach, whose validity is supported by Monte Carlo simulations [23] and experimental investigations [22].

Within the BioQuaRT project, measurements are carried out in which two of the nanodosimeters are used to measure the same radiation quality of carbon ions as provided by the ion accelerator facilities of Warsaw University Heavy Ion Laboratory (HIL, Poland) [24] and of the National Nuclear Physics Institute Laboratories at Legnaro (LNL, Italy). To this end, the Jet counter has been modified and relocated to HIL, and the PTB Ion Counter has been significantly modified to make it transportable for operation at HIL, LNL and PTB. In addition, the detection scheme of the PTB Ion Counter has been redesigned to allow the use of alternative detectors for the primary particles. Using a new position sensitive primary particle detector, arbitrary beam conditions can now be simulated (see Figure 2).

A total of four measurement campaigns have already been conducted for comparing the PTB Ion counter with 
the other nanodosimeters by measuring the same radiation qualities of carbon beams [25, 26]. The purpose of these comparisons is to investigate the correlation between nanodosimetric distributions of ionisation cluster size for different simulated target size as well as different impact parameter of the particle track with respect to the target volume. Furthermore, the Jet Counter's unique capability of recording ionisation cluster size distributions created by electrons has been pushed toward the nanodosimetric characterisation of delta electrons by measuring ionisation cluster size distributions produced by the Auger emitting nuclide ${ }^{125} \mathrm{I}[27]$.

A further goal of the nanodosimetry work package is a prototype experimental multi-scale characterisation of the track structure for the ion beams that are used in the radiobiological studies in work package 4. For this goal, the usability of a silicon micro-telescope [13] for primary particle detection allowing simultaneous measurement of lineal energy and ionisation cluster size has been demonstrated. Furthermore, the possibility to measure with gas mixtures simulation DNA material composition has also been successfully tested.

\subsection{Indirect Effects}

Indirect damage to DNA caused by ionising radiation is the result of the radiolysis of water and a complex subsequent chain of chemical and biochemical reactions $[4,28]$. The vast literature of radiation chemical yield of biologically significant reactive species in aqueous environments has been reviewed and a data base has been produced that will be made accessible through the BioQuaRT web site [2]. Particular focus lay on the effect of radiation type and energy on the relative amounts and spatial distribution of different species produced with the goal of identifying specific probes and techniques best suited to determine the relative amounts and distributions of species produced and to relate these quantitatively to biological effectiveness.

Further work directed at investigating options for characterising the spatial distribution of biologically significant reactive species in aqueous environments has been started selecting coumarin as the initial probe. This was chosen because of the known specific reaction of coumarin-3-carboxylic acid with $\mathrm{OH}$ radicals to form a stable fluorescent product, which can be detected with high sensitivity. Feasibility of direct imaging methods and characterisation by magnetic resonance techniques (EPR, NMR), that characterise the environment surrounding radiation induced species [29], will be theoretically evaluated and a test system will be developed based on a selected method.

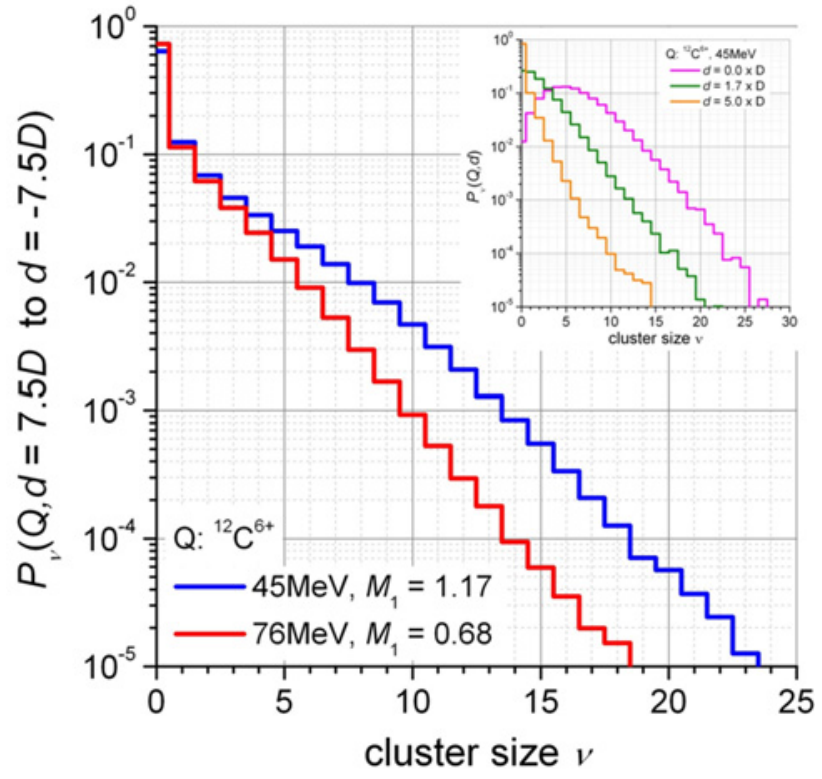

Figure 2. Broad beam ionisation cluster size distributions measured with the PTB Ion Counter nanodosimeter for two radiation qualities of carbon ions. Inset: Ionisation cluster size distributions for $45 \mathrm{MeV}$ carbon ions and different distance $d$ (in multiples of the target diameter $D$ ). (reproduced from [24]).

\subsection{Radiobiology}

In this work package, biological experiments on cultured cells are conducted based on a known number of particle tracks (traversing the cell or the cell nucleus) to provide a set of biological data to use as benchmarks for the predictions of the multi-scale model that is developed in WP5.

These biological data comprise three different biological endpoints: First, the formation of $\gamma-\mathrm{H} 2 \mathrm{AX}$ foci [29], which occur within minutes at loci of (initial) DNA double strand breaks (DSBs) and disappear when these DSBs are removed. The other two endpoints are the induction of micronuclei [31] and of dicentric chromosomes (see for example a recent review by Rothkamm et. al. [32]). These two biological endpoints can be observed as a consequence of the partial failure of the cell to repair the initial DNA damage induced by the irradiation.

Measurements performed so far used the PTB ion microbeam where accelerated protons and $\mathrm{He}$ ions are available over a large range of linear energy transfers (LET). Working protocols for the irradiation of human cell types, Human Umbilical Vein Endothelial Cells (HUVECs) and Normal Human adult dermal Fibroblasts (NHDF), and the subsequent measurement of $\gamma$-H2AX foci have been established, as well as working protocols to quantify dicentric chromosomes and micronuclei in Chinese Hamster Ovary (CHO) cells. Irradiations with protons accelerated to an energy of $3 \mathrm{MeV}$ and $10 \mathrm{MeV}$, and alpha particles of $8 \mathrm{MeV}$ and $20 \mathrm{MeV}$ were conducted, featuring linear energy transfers (LET) of $18 \mathrm{keV} / \mu \mathrm{m}, 5$ $\mathrm{keV} / \mu \mathrm{m}, 160 \mathrm{keV} / \mu \mathrm{m}$ and $37 \mathrm{keV} / \mu \mathrm{m}$, respectively. 


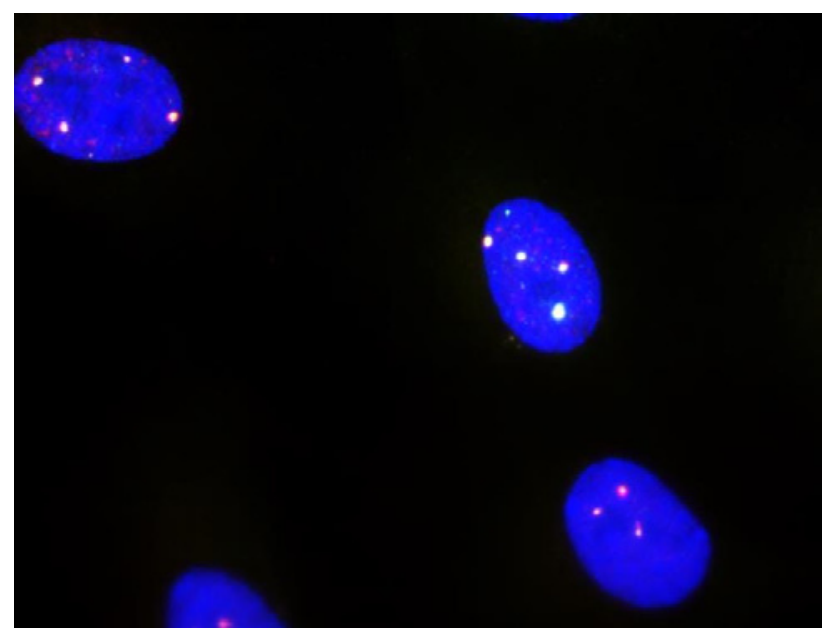

Figure 3. Micrograph of human fibroblasts. The nucleus of each cell (stained in blue) was targeted by five $20 \mathrm{MeV}$ alpha particles. After the irradiation, biological assays were performed to detect induced DNA double strand breaks through the $\gamma-\mathrm{H} 2 \mathrm{AX}$ foci (bright spots).

Earlier irradiation runs conducted during the first part of the project have been tailored to the set-up of all the biological assays at the PTB ion microbeam, particularly those to measure "late" effects of ionising radiation, that is those effects that become apparent after cells have attempted, and possibly failed, to restore the correct genomic integrity after the radiation insult (micronuclei and dicentric chromosomes among them). While typical microbeam irradiations for "early effects experiments", such as those for measurement of $\gamma-\mathrm{H} 2 \mathrm{AX}$ foci, involve relatively small number of viable cells, "late effects" assays necessitate a relatively large number of viable cells and this has required a series of experiments to make the experimental work sustainable.

Results from all biological experiments conducted at the PTB ion microbeam will be presented in future, dedicated reports.

\subsection{Multi-scale model}

Work package 5 is intended to assemble different results of the already presented work packages in a global model that allows predicting the biological damage to cells depending on the radiation quality.

From a practical point of view, the deliverable of this work package is a software code that can be used to simulate ionising radiation interaction with biological matter at cellular (micrometric) and subcellular (nanometric) scale. This multi-scale code should give the relevant information on the energy deposition at both scales in the target geometry that allows characterising, differentiating and predicting the biological effects of the different radiation qualities starting from the physical irradiation parameters.

The simulation of the initial interactions (physical stage) between the ionising radiation and the biological target is performed using Monte Carlo simulation techniques. This method requires interaction crosssections between the projectile and the target material. The first task in work package 5 provides evaluated data sets of cross-sections for the interaction of charged particles with ingredients of the DNA that are obtained at PTB [33, 34] to be used in the Monte Carlo codes. The cross sections for electron scattering on some of the DNA constituents have already been implemented into the PTB track structure code PTra. Simulations based on this augmented code indicate that using only cross section of water in track structure simulations may lead to an underestimation of radiation damage to the DNA (see Figure 4).

While the determination of a comprehensive cross section data set for radiation interaction with all DNA constituents for all particles of interest is still in progress, calculations have already been performed, using liquid water cross-sections, in order to study and determine the parameters allowing the correlation of the energy deposition at micrometric and nanometric scale depending on the radiation quality. The Monte Carlo code Geant4DNA [35], was used to simulate proton, alpha particle and carbon ion tracks to obtain the microdosimetric and nanodosimetric distributions characterising the track structure for a large range of linear energy transfers. Besides the conventional homogeneous micrometric target also a more realistic geometrical model representing the chromatin structure of the DNA was used [36].

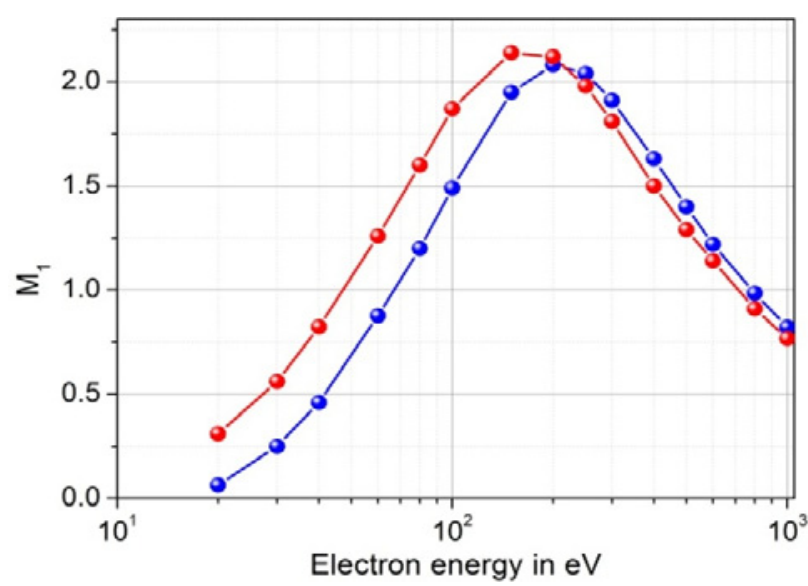

Figure 4. Simulation results for the mean number of ionisations produced by monoenergetic electrons in a cylindrical target volume having the size of one convolution of the DNA double helix. The target volume was surrounded by liquid water and filled with liquid water (blue symbols) and a mixture of DNA constituents (red symbols) at unit density, respectively.

A first attempt was made to correlate microdosimetric distributions and nanometric clusters of ionisation or energy deposits which can be understood in terms of the secondary electron spectra for the different radiation qualities [37]. The results obtained using liquid water material will be consolidated by using the augmented Monte Carlo codes with the experimental DNA crosssections and the DNA target structure. Results of work package 2 will contribute to this validation by giving information about the microdosimetric spectra of the radiations in coincidence with the ionisation clusters size produced at nanometric scale.

The simulation of both, the physical and the chemical stage of the irradiation are needed in the model in order to account for total (direct and indirect) early effects on DNA. Starting from the simulation of the chemical stage 
available in the Geant4-DNA package, results on the spatial distribution of radiation-induced radicals obtained in work package 3 will be included to improve this model.

All the aforementioned data and simulation tools are used in this work package to simulate (at nanometric scale) the microbeam irradiations being performed in work package 4. The initial objective is to identify and determine the parameters in the simulation that are significant for a correlation to the experimental $\gamma-\mathrm{H} 2 \mathrm{AX}$ results. The outcome of these early radiation-induced damages in terms of late effects will enable the realisation of a software tool for the prediction of the biological consequences of charged particle irradiation.

\section{Researcher Grants}

As mentioned before, three so-called Researcher Excellence Grants (REGs) are linked to the joint research project. One of these REGs is directly integrated into BioQuaRT while the other two address issues that go beyond the scope of this project.

The research activities of the first REG are part of BioQuaRT work packages 1 and 2, i.e. microdosimetric and nanodosimetric characterisation of proton and carbon ion beams, for which the researcher inter alia designed a setup for joint measurements with micro-calorimeters, mini-TEPC and Si-based microdosimeters in high-energy ion beams with adjustable positions along the depth-dose curve of a Bragg peak.

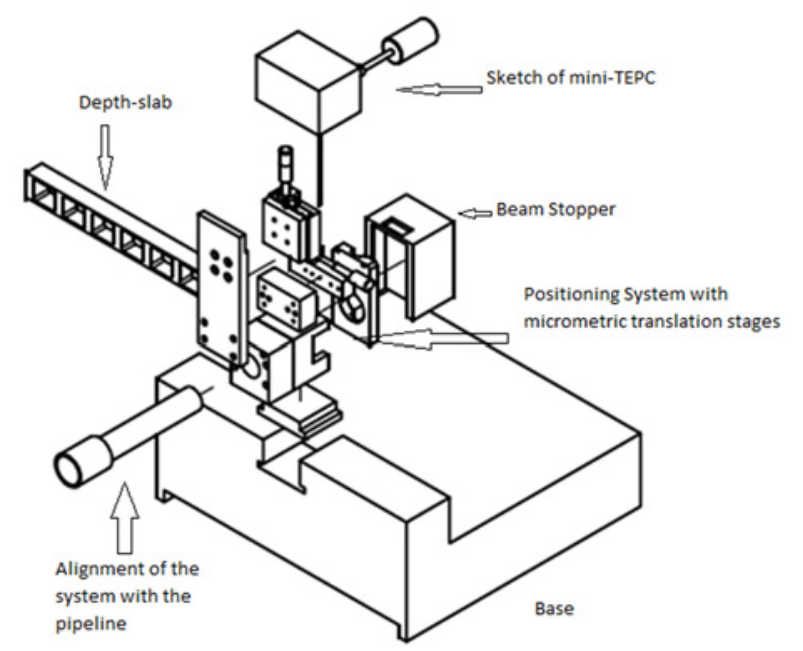

Figure 5. Schematic assembly drawing of the set-up designed by the REG(INFN) researcher for comparison of different microdosimeters in the same high energy beam for different position along the Bragg peak of protons and carbon ions.

This REG researcher works at Laboratori Nazionali di Legnaro of INFN within the radiation physics group that has a long-standing expertise in microdosimetry and nanodosimetry. This group is leader of the INFN-funded Italian experiment named MITRA, which performs microdosimetric and nanodosimetric measurement of different light ions beam (e.g. lithium, helium, boron in addition to carbon ions and protons) with different detector types [38]. As such, MITRA and BioQuaRT are complementary.
The second REG, awarded to the university hospital MRI of Technical University Munich, started in June 2013 and develops methods which allow a translation of novel dosimetric quantities derived from particle track structure to clinical applications in ion beam therapy.

The third REG researcher works at Lisbon Technical University institute IST-ID. Started in November 2013, the research develops and experimentally benchmarks a new biophysical model for induction of early radiation damage and resulting cell killing.

In addition to the REGs, an early stage researcher mobility grant has been awarded to a PhD student from the Belgium nuclear research institute SCK.CEN for extended research stays with INFN at Legnaro to investigate means for optimising the response of mini tissue-equivalent proportional counters in therapeutic proton and carbon ion beams.

\section{Dissemination}

A project web site has been established to inform interested stakeholders on the progress of this joint effort towards realising a comprehensive and systematic investigation of the links between the physical distribution of ionisation and the biological effects of ion beam radiation qualities [2].

In addition, the project organises three stakeholder dissemination workshops. The first one was held as a satellite workshop of the $12^{\text {th }}$ Neutron and Ion Dosimetry Symposium (NEUDOS-12) on 7 June 2013 in Aix-enProvence, France. The programme included presentations on the five work packages as well as from the MITRA project [31] and the Marie Curie Action ARDENT [39]. All presentations have been posted on the BioQuaRT web site [40].

The second workshop will take place at the Austrian ion beam therapy facility MedAustron on 7-9 May 2014 [41]. The final dissemination workshop is planned to be incorporated into the 34th meeting of the European Society for Therapeutic Radiology and Oncology (ESTRO 34) in spring 2015 [42].

\section{References}

1. http://www.emrponline.eu/

2. http://www.ptb.de/emrp/bioquart.html

3. S. M. Seltzer (Ed.), ICRU Report No. 85, Journal of the ICRU 11, 1-32 (2011)

4. C. von Sonntag, The chemical basis of radiation biology, Taylor \& Francis, London, (1987)

5. H. H. Rossi, M. Zaider, Microdosimetry and its Applications, ISBN 3-540-58541-9, Springer, Heidelberg, (1996)

6. B Großwendt, Radiat. Prot. Dosim. 110, 789-799 (2004)

7. R. W. Schulte, AIP Conf. Proc. 1345, 249-261 (2011)

8. S. Galer, L. Hao, J. Gallop, H. Palmans, K. Kirkby and A. Nisbet, Radiat. Prot. Dosim. 143, 427-431 (2011)

9. S. Galer, Development of a Microbolometer for Microdosimetry of Ionising radiation (PhD Thesis, University of Surrey, 2012) 
10. L. Hao, J. C. Gallop, C. Gardiner, P. Josephs-Franks, J. C. Macfarlane, S. K. H. Lam and C. Foley, Supercond. Sci. Technol. 16, 1479-1482 (2003)

11. K. Fathi, S. Galer, H. Palmans, J. Gallop and K. J. Kirkby, $11^{\text {th }}$ Microbeam Workshop, Bordeaux, France 3-4 October 2013

12. S. Agosteo, P. G. Fallica, A. Fazzi, M. V. Introini, A. Pola and G. Valvoc, Radiat. Meas. 43, 585-589 (2008)

13. S. Agosteo and A. Pola, Radiat. Prot. Dosim. 143, 409-415 (2011).

14. L. De Nardo, V. Cesari, G. Donà, G. Magrin, P. Colautti, V. Conte and G. Tornielli, Radiat. Prot. Dosim. 108, 345-352 (2004)

15. L. De Nardo, D. Moro, P. Colautti, V. Conte, G. Tornielli and G. Cuttone, Radiat. Prot. Dosim. 110, 681-686 (2004)

16. S. Agosteo, G.A.P. Cirrone, P. Colautti, G. Cuttone, G. D’Angelo, A. Fazzi, M.V. Introini, D. Moro, A. Pola and V. Varoli, Radiat. Meas. 45, 1284-1289 (2010)

17. S. Pszona, J. Kula, and S. Marjanska, Nucl. Instrum. and Meth. in Phys. Res. A 447, 601-607 (2000).

18. A. Bantsar, B. Grosswendt, and S. Pszona, Radiat. Prot. Dosim. 122, 82-85 (2006)

19. L. De Nardo, A. Alkaa, C. Khamphan, V. Conte, P. Colautti, P. Ségur, G. Tornielli, Nucl. Instrum. Meth. A 484, 312-326 (2002)

20. V. Conte, P. Colautti, L. De Nardo, A. Ferretti, M. Poggi, D. Moro, M. Lombardi, G. Tornielli and B. Grosswendt. Radiat. Prot. Dosim. 143, 455-358 (2011)

21. G. Garty, S. Shchemelinin, A. Breskin, R. Chechik, I. Orion, G.P. Guedes, R. Schulte, V. Bashkirov, B. Grosswendt, Radiat. Prot. Dosim. 99, 325-330 (2002)

22. G. Hilgers, Radiat. Meas. 45, 1228-1232 (2010).

23. B. Grosswendt, Radiat. Prot. Dosim. 99, 401-404 (2002)

24. http://www.slcj.uw.edu.pl/en/

25. A. Bantsar, G. Hilgers, S. Pszona, H. Rabus and Z. Szeflinski, Proc. MICROS-16 conference, Treviso, Italy, 20-25 October 2013, to appear in Rad. Prot. Dosim. (2014)

26. D. Moro, P. Colautti, V. Conte, G. Hilgers, A. Pausewang, W. Helms, B. Lambertsen, H. Rabus, LNL-INFN Annual Report (ISSN 1828-8545) 239, 178-179 (2013).

27. S. Pszona, B. Grosswendt, A. Bantsar, I. Cieszykowska, W. Czarnacki, Radiat. Meas. 47, 1092-1096 (2012)

28. J. A. LaVerne, Track Effects of Heavy Ions in Liquid Water, Radiation Research 153, 487-496 (2000)

29. M. Marrale, A. Longo A. Barbon, M. Brustolon, M. Brai, Proc. NEUDOS-12 conference, Aix-enProvence, France, 3-7 Jun 2013, to appear in Rad. Prot. Dosim. (2014)

30. E. P. Rogakou, C. Boon, C. Redon, W. M. Bonner, The Journal of Cell Biology 146, 905-916 (1999)

31. M. Fenech, Mutat Res 455, 81-95 (2011)

32. K. Rothkamm et al., Radiat. Res. 180, 111-119 (2013)

33. W. Y. Baek, M. Bug, H. Rabus, E. Gargioni, and B. Grosswendt, Phys. Rev. A 86, 032702 1-15 (2012), DOI: 10.1103/PhysRevA.86.032702
34. W. Y. Baek, A. Arndt, M. Bug, H. Rabus, M. Wang, Phys. Rev. A 88, 032702 1-8 (2013), DOI: 10.1103/PhysRevA.88.032702

35. S. Incerti et al., Med. Phys. 37, 4692-4708 (2010)

36. M. Dos Santos, C. Villagrasa, I. Clairand and S. Incerti, Nucl. Inst. and Meth. in Phys. Res. B 298, $47-$ 54 (2013)

37. D. Bianco, C. Villagrasa and M. Dos Santos, NEUDOS-12 conference, Aix-en-Provence, France, 3-7 Jun 2013

38. http://www.lnl.infn.it/ microdos/MITRA.html

39. http://ardent.web.cern.ch/ardent

40. http://www.ptb.de/emrp/bioquart_neudos12.html

41. http://www.ptb.de/nanocord/mind-ibct.html

42. http://www.estro.org/congresses-meetings/next-event 